\title{
RELACIÓN ENTRE LOS COMPORTAMIENTOS ASOCIADOS CON EL TRASTORNO POR DÉFICIT DE ATENCIÓN E HIPERACTIVIDAD Y LOS ESTILOS DE CRIANZA DESDE LA APRECIACIÓN DE LOS ADULTOS
}

\section{Relationship between behaviors associated with Attention deficit hyperactivity disorder (ADHD) and the upbringing styles from the appreciation of adults}

Jahaziel Molina Del Rio*, Deyanira Arellano Martínez**; Víctor Hugo

González Becerra***; Claudia María Ramos Santana****

Centro Universitario de los Valles de la Universidad de Guadalajara, México

\section{Resumen}

El presente estudio propone caracterizar la relación entre los estilo de crianza de padres y las conductas relacionadas con TDAH. Para ello se requirió la participación de 46 padres de los niños pertenecientes a la escuela primaria Juan A. Mateos, a los cuales se les solicitó contestaran la Adaptación del Cuestionario de Crianza Parental (PCRI-M), y la encuesta de criterios de diagnóstico de trastorno por déficit de atención e hiperactividad.

Se realizó un análisis X2 de los datos obteniendo para las variables de estilo de crianza y sospecha de TDAH referida por los adultos (padres y profesores), sugiriendo una relación entre el estilo de crianza autoritario y la no sospecha de TDAH por parte de padres, mostrándose también según la apreciación de los profesores una relación entre el estilo de crianza permisivo y la sospecha de TDAH.

Palabras clave: TDAH, estilos de crianza, estilos parentales, desatención, hiperactividad, permisivo, autoritario, disciplinado.

\section{Abstract}

The present study proposes to characterize the relationship between upbringing styles and $A D H D$ related behaviors. This involved the participation of 46 parents of children belonging to the elementary school Juan A. Mateos, who were asked to respond to the Adaptation of the Parenting Questionnaire, and the survey of diagnostic criteria for Attention deficit disorder and hyperactivity. We performed an X2 test of the data obtained for the variables of upbringing style and suspected ADHD referred by adults (parents and teachers), suggesting a relationship between authoritarian parenting style and non-suspected ADHD by parents, also showing according to the appreciation of teachers a relationship between permissive parenting style and suspected ADHD.

Keywords: ADHD, upbringing styles, parenting styles, inattention, hyperactivity, permissive, authoritarian, disciplined.

\footnotetext{
* Psicólogo. Docente Centro Universitario de los Valles de la Universidad de Guadalajara. jahaziel. molina@valles.udg.mx

** Estudiante de la Licenciatura en Psicología del Centro Universitario de los Valles de la Universidad de Guadalajara

*** Psicólogo. Docente Centro Universitario de los Valles de la Universidad de Guadalajara.

**** Psicóloga. Docente Centro Universitario de los Valles de la Universidad de Guadalajara.
} 


\section{INTRODUCCIÓN}

El Trastorno por Déficit de Atención e Hiperactividad (TDHA) es un trastorno que afecta a la población en edad infantil a nivel mundial, tan solo en Latinoamérica esta afectación se diagnosticó a 36 millones de personas y, específicamente, en México se encontró en 1.5 millones de niños de entre seis y doce años (Huesca, 2008), la prevalencia a nivel mundial del trastorno se considera del $16.1 \%$ cuando se tiene en cuenta solamente los síntomas y aproximadamente del $6.5 \%$ cuando se incluye el criterio de afectación funcional para su diagnóstico (Cáceres y Herrero, 2011).

El TDAH representa un problema crónico de salud pública, ya que llega a tener efectos significativos en el funcionamientos de los niños y adultos en múltiples áreas (Cardo, De Azua, Redondo, Riutort, Severa, y Vidal, 2011).

El estudio sobre el TDAH ha dado de manifiesto diferentes características del trastorno, entre las que se encuentran las neuroanatómicas (Almeida, 2005; Almeida, Ricardo-Garcel, Prado y Martínez, 2010), electrofisiológicas (Cornelio-Nieto, Borbolla-Sala, Gallegos-Dimas, 2011; Escobar, Schmidt y Sardinas, 2011; Clarke, Barry, McCarthy y Selikowitz, 2001), farmacológicas (Mulas, Gandía, Roca, Etchepareborda y Abad, 2012; Castellanos y Acosta, 2011) y cognitivas (Johnstone y Clarke, 2009; Heatherton y Wagner, 2011, Stern y Shalev, 2013). Estas últimas se centran en aspectos particulares como el autocontrol sobre el habla, la cual se caracteriza por ser excesiva, presentar interrupción del discurso del otro, no esperar turno, hacer comentarios fuera de lugar, e iniciar conversaciones en momentos poco adecuados (Calvo, Herrera y Peyres, 2003); así como afectaciones en diferentes funciones, debido a la hiperactividad/ impulsividad que engloban dificultades en procesos de percepción, atención sostenida, integración, memoria de trabajo, capacidad de cambio de foco atencional, planificación y persistencia en la acción (Cáceres y Herrero, 2011).

En los últimos años se ha demostrado que el TDAH afecta considerablemente el bienestar de los niños y adolescentes, así como en su capacidad para el funcionamiento diario, encontrando que los niños con TDAH tienen un panorama disfuncional en los siguientes dominios: impacto emocional sobre lo padres, comportamiento, autoestima y funcionamiento psicosocial (Colomer-Diago, Miranda-Casas, Presentación-Herrero, y Rosello, 2011). La presencia de este trastorno en la etapa de la niñez incrementa el riesgo de presentar accidentes, fracaso escolar, tendencia a ser agresivos, destructivos y dominantes (De la Peña, Palacio y Barragán, 2010).

Las personas con TDAH evitan las actividades donde se requiere de atención sostenida, dedicación personal, organización, concentración, etc. Debido a la incapacidad de mantener su atención orientada a un estímulo por periodos prolongados de tiempo. En actividades sociales y cotidianas suelen aparentar no escuchar lo que se les dice, por lo que ignoran o realizan cambios frecuentes en la conversación, son olvidadizos y se distraen fácilmente con estímulos que para lo demás son irrelevantes, como sonidos, ruidos, conversaciones ajenas, etc. (Correas, Quintero y Quintero, 2009).

El TDAH predominantemente se identifica desde la niñez, por lo que el diagnóstico es más frecuente en la edad escolar, según Cáceres y Herrero (2011) el número de niños con diagnóstico de TDAH es significativamente superior en comparación al número de niñas, siendo más común los casos en niños entre las edades de 6 y 10 años y entre los 11 y 15 años, a diferencia de las niñas que se manifiesta más entre los 16 y 20 años.

Aun con los diferentes datos que han caracterizado el TDAH, los principales criterios para su diagnóstico siguen siendo los establecidos en el Manual Diagnóstico y Estadístico de los Trastornos Mentales (DSM por sus siglas en inglés) (APA, 2013), los cuales han sido retomados para la formación de instrumentos diagnósticos (Farré y Narbona, 2013), en los que la principal fuente de información de las conductas del TDAH son los padres, quienes por medio de una serie de preguntas basadas en los criterios del DSM, califican la frecuencia de la presencia de los síntomas, brindando como resultado un posible diagnóstico del trastorno.

Se ha hecho hincapié en que la presencia de este tipo de conductas debe existir en al menos dos escenarios: 
casa, escuela, parques, etc. (De la Peña, Palacio y Barragán, 2010), de igual forma se considera que estos síntomas deben presentar un impacto negativo en la vida de los niños, lo que produce un impacto social que se vincula al funcionamiento del individuo en su entorno familiar, escolar y en relaciones con sus pares, ya que la sola presencia de los síntomas no refiere que se padece del trastorno (Abad y cols., 2012). Aspectos que no siempre son considerados en los instrumentos de evaluación.

De tal manera que existe una relación bidireccional entre el niño y su entorno, la cual no siempre es recíproca, entendiendo esto como aquellas ocasiones en que las acusaciones de conductas por parte de los adultos parecen indicar una mayor prevalencia del trastorno, mientras que si se consideran los componentes de afectación funcional este índice se reduce (Cáceres y Herrero, 2011).

Galimberti (2006) refiere que durante los primeros siete años de vida se ha desarrollado una atención predominantemente espontánea, pero a partir de los ocho a los once años los niños son capaces de orientar la atención hacia un objeto particular, asociado a la aparición de la atención voluntaria.

El factor social juega un rol importante en el proceso atencional, no sólo para su diagnóstico sino también para su desarrollo, desde la psicología clásica Vygotsky (1986) concibe gran importancia al rol de los adultos en la adquisición y consolidación de la atención, ya que estos regulan la atención voluntaria de los niños, guiándola mediante instrucciones verbales que se caracterizan por ser activas y conscientes, las cuales posteriormente los niños interiorizan adquiriendo y consolidando así el control de los procesos atencionales.

Esta concepción del medio familiar como un regulador y formador de la conducta ha sido considerada como un punto principal en la intervención del trastorno, como ha quedado de manifiesto en el inciso 20 de la declaración de Cartagena que a la letra señala "La familia es la responsable del tratamiento de su hijo, para lo cual debe recibir todo el apoyo técnico y material que necesite" (De la Peña, Palacio y Barragán, 2010 pp. 96), considerando también la relevancia del medio ambiente como modulador en la expresión del trastorno.

Dentro del medio ambiente, el medio familiar juega un rol importante en el desarrollo de las personas, el conjunto de acciones que ejercen los padres sobre los hijos como los principales transmisores de principios, conocimientos, valores, actitudes, roles y hábitos que de una generación pasa a la siguiente son considerados como estilos de crianza (Navarrete, 2011). De esta forma se van formando normas en la familia, las cuales los padres o cuidadores primarios pretenden hacer cumplir (Mestre, Samper, Tur y Díez, 2001). Las prácticas educativas de los padres pueden estar determinadas por distintos factores, estas pueden dividirse en tres grupos: las que están relacionadas con los niños, las relacionadas a los padres y aquellas que se relacionan a la situación en donde se lleva a cabo la interacción (Palacios, 1988; Ramírez, 2005). Los estilos de crianza son la forma de actuar de los adultos sobre el niño diariamente y en todo momento, por ejemplo, en toma de decisiones o resolución de conflictos, lo cual ayuda a que el niño regule sus conductas así como también marcan los límites, que serán relevantes en el desarrollo del menor (Torío, Peña y Rodríguez, 2008).

Diana Baurind (1966) clasificó 3 estilos de crianza: a)crianza autoritaria, donde la palabra de los padres es ley, por lo que los hijos no tienen oportunidad de opinar o poner en discusión el asunto, existe un castigo hacia la mala conducta, generalmente es un castigo físico, los padres autoritarios tienen reglas claras, no permiten que sus hijos expresen opiniones o sentimientos, por lo que los padres no escuchan a sus hijos, y los padres no suelen mostrar conductas de afecto hacia ellos; b)crianza permisiva, estos padres tiene pocas exigencias hacia sus hijos, no expresan impaciencia hacia ellos, por lo que existe poca disciplina y en consecuencia exigen escasa madurez en los niños, este tipo de padres son muy cariñosos ante sus hijos, por lo que los escuchan y tratan de ayudarles en todo, pero no se sienten responsables de su conducta, los padres de estos niños se mostraban indiferentes ante las actitudes y conductas del niño, tanto positivas como negativas, además de caracterizarlos como pasivos y permisivos, ya que evitan ser la autoridad evadiendo castigos y permitiendo todos los impulsos de su hijos (Torío, 
Peña y Rodríguez, 2008); c)crianza disciplinada, donde los padres establecen normas y límites, pero también escuchan las demandas de sus hijos, estos padres exigen madurez pero a su vez son cariñosos, comprensivos, y habitualmente perdonan y no castigan, ya que estos padres son flexibles y escuchan el argumento de los hijos (Stassen, 2007).

El estilo de crianza de los padres determinará ciertas conductas y actitudes que manifiestan los niños en su desarrollo. Se ha demostrado que los niños presentan características similares al estilo de crianza que ejercen sus padres, Stassen (2007) nos menciona que los niños criados de padres autoritarios tienden a ser conscientes, obedientes y pasivos, pero no son felices, constantemente se sienten culpables o infelices, internalizan sus frustraciones y se culpan cuando las cosas no salen bien. Los niños que son criados por padres permisivos son aún menos felices, estos niños carecen de autocontrol, ya que el padre no punitivo acepta los impulsos del niño, en especial si se refiere a dar y recibir entre sus pares, son inmaduros y les cuesta trabajo hacer amistades (Baumrind, 1966; Mestre y cols., 2001). Los padres disciplinados crían hijos con más probabilidades de ser coherentes, inteligentes, felices con ellos mismos por lo que son niños apreciados por lo que los rodean. Además de que si los padres prestan atención, cuidados y exigen niveles de control adecuados a la edad y desarrollo que se encuentran se fomenta en lo niños madurez y competencia.

En una investigación cuyo objetivo era mostrar las características que los niños desarrollan según el estilo de crianza de los padres, se entrevistó a niños de familias disciplinados observando que presentaban elevadas competencias sociales y cognitivas, niños de familias autoritarias mantenían un nivel medio e hijos de familias permisivos o negligentes presentaban los niveles más bajos de dichas competencias, manteniendo estas actitudes hasta la adolescencia, (Torío, Peña y Rodríguez, 2008).

Aunque la influencia del ambiente familiar no es considerada como un aspecto que origina los síntomas del trastorno, no solo es considerada relevante para su expresión si no para su diagnóstico, ya que en muchas de las pruebas para su diagnóstico, la principal fuente de información es referida por los padres, quedando a expensas de la referencia subjetiva que ellos perciban de las conductas de sus niños.

Es por eso que nos interesamos en conocer si el estilo de crianza que ejercen los padres hacía con sus hijos presenta alguna relación con el reporte que hacen de las conductas evaluadas en el TDAH, considerando el criterio diagnóstico por parte de los profesores y los propios padres. Por lo que nuestro objetivo fue caracterizar la relación entre los comportamientos asociados al TDAH, apreciados por los adultos y los estilos de crianza de los padres.

\section{MÉTODO}

\section{Sujetos}

Se entrevistaron a los padres de familia y profesores de 46 alumnos de los grados de $4^{\circ}, 5^{\circ}$ y $6^{\circ}$ de la escuela primaria Juan A. Mateos de la ciudad de Guadalajara, Jalisco, México. Los padres de familia fueron seleccionados por muestreo no probabilístico, siendo considerados sólo aquellos padres y profesores que desearon participar de manera voluntaria contestando las encuestas, tomado en cuenta a aquellos que pasaban el mayor tiempo al cuidado de sus hijos.

\section{Instrumentos}

Para la recolección de datos se utilizaron dos instrumentos, la Adaptación del Cuestionario de Crianza Parental (PCRI-M) (Barrio y Roa, 2001), y la encuesta de criterios diagnósticos del TDAH de la prueba NEUROPSI Atención y Memoria (Ardila y cols., 2012).

El PCRI-M consiste en 78 preguntas cuyos ítems se dividen en apoyo, satisfacción a la crianza, compromiso, comunicación, disciplina, autonomía, distribución del rol y deseabilidad social. Con estos apartados se obtiene un puntaje para determinar el estilo de crianza de los padres. El estilo de crianza se puede clasificar en alguna de las siguientes categorías: a) autoritaria cuando en todas las áreas presenta puntuaciones bajas, b) permisivo si son elevadas en las escalas de apoyo y bajas en las escalas de disciplina $\mathrm{y}, \mathrm{c})$ disciplinada si las puntuaciones son altas en todas las áreas. 
La encuesta de criterios de diagnóstico de TDAH (apartado de la prueba de NEUROPSI). Este cuestionario debe ser contestado por padres o profesores que refieran conductas de personas entre los 6 y 17 años. El cuestionario consta de dos modalidades una para padres y otra para profesores. Consiste en 18 preguntas que se dividen en dos apartados, el primero consta de 9 preguntas relacionadas a síntomas de inatención y el segundo que consta de 9 preguntas relacionadas a hiperactividad-impulsividad, las respuestas a estas preguntas se clasifican y califican según su frecuencia en "nunca" con cero puntos, "algunas veces" con un punto, "muchas veces" con dos puntos y "casi siempre" con tres puntos. La puntuación se obtiene identificando aquellas preguntas calificadas con una frecuencia correspondiente a dos o tres, si la sumatoria corresponde a un número igual o mayor a 6 preguntas con estas frecuencias se interpreta como una sospecha de TDAH.

\section{Procedimiento}

En la etapa inicial se presentó el proyecto y se pidió la autorización a los directores de la escuela de realizar una investigación con los grupos de $4^{\circ}, 5^{\circ}$ y $6^{\circ}$ grado de primaria del turno matutino. Después se les notificó a padres de familia de la investigación que se realizaría, y se solicitó su participación de manera voluntaria, a aquellos padres que decidieron participar se les pidió la firma del consentimiento informado.

Posteriormente, se les entregó a los padres de familia dos juegos de cuestionarios, el primero era la encuesta para padres de los criterios del TDAH que forma parte del NEUROPSI, y el segundo el PCRI$\mathrm{M}$, se les solicitó a los padres que contestaran los cuestionarios en casa durante algún momento que pudieran dedicarle tiempo y atención, pidiéndoles que en un plazo no mayor a una semana regresarán los cuestionarios contestados por medio de sus hijos, quienes debían entregarlo al profesor para que este último los entregue al investigador.

De igual manera se entregó a cada profesor encargado de grupo los ejemplares necesarios de la encuesta de los criterios del TDAH del NEUROPSI, se le solicitó respondiera uno por cada uno de los alumnos de su grupo.
Una vez que se recabó el material, se calificaron las encuestas de padres y profesores del NEUROPSI y las encuestas del PCRI-M, los datos se agruparon y analizaron con el Paquete Estadístico para la Ciencias Sociales (SPSS por sus siglas en inglés) versión 20 (IBM, 2011), aplicando estadística descriptiva y la prueba X2 para evaluar la dependencia de las variables estilo de crianza y sospecha de TDAH, considerando un valor p igual o menor a 0.05 como estadísticamente significativo.

\section{Resultados}

Del total de los 46 padres evaluados, 16 tienen hijos en cuarto grado, 18 en quinto grado y 12 en sexto grado. Se recuperaron un total de 28 cuestionarios del PCRI-M, 27 de las encuestas de criterios de diagnóstico del TDAH de los padres y 43 encuestas de criterios de diagnóstico de TDAH de los profesores.

Los resultados del PCRI-M muestran la prevalencia de los estilos de crianza que presentan los padres evaluados, de los cuales el $4 \%$ correspondió al estilo de crianza disciplinado, $64 \%$ de los padres reportaron mantener un estilo de crianza autoritario y $32 \%$ de los padres reportaron un estilo de crianza permisivo (ver figura 1).

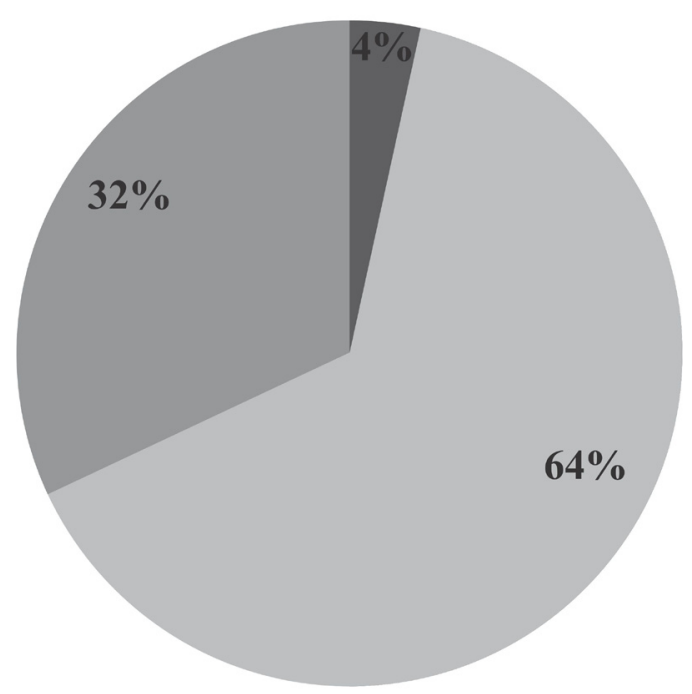

Disciplinado Autoritario Permisivo

Figura 1. Distribución del porcentaje de estilos de crianza reportado por los padres. 
En cuanto a la encuestas de criterios de diagnóstico del TDAH se observó que el $11 \%$ de los padres de familia refirieron que sus hijos presentan conductas con la frecuencia suficiente para ser consideradas como sospecha de TDAH, mientras que el $89 \%$ no lo reporta así (ver Figura 2).

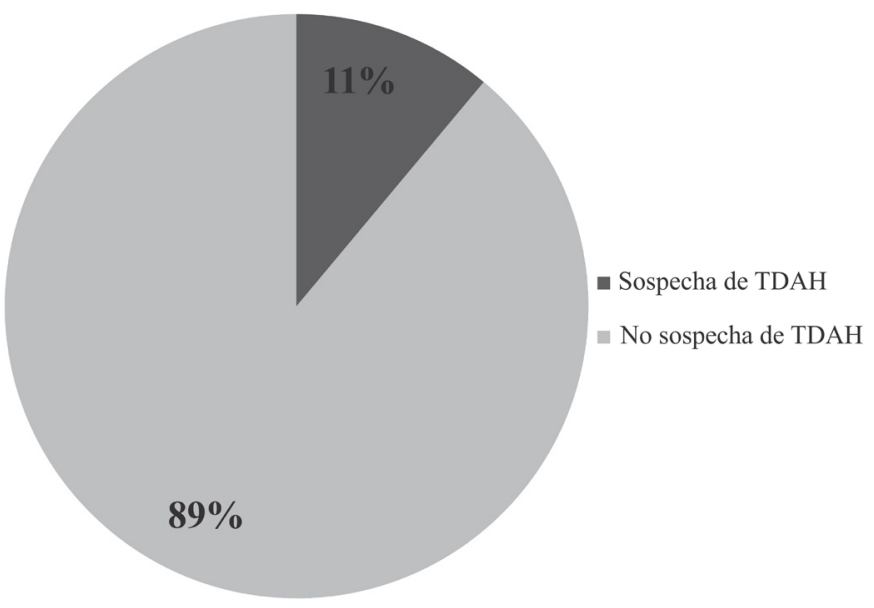

Figura 2. Distribución del porcentaje de casos con y sin sospecha de TDAH por parte de los padres.

Mientras que el porcentaje de casos de niños que presentan conductas con la frecuencia suficiente para ser consideradas como sospecha de TDAH juzgadas por parte de los profesores es del 37\% de los casos y en un $63 \%$ de ellos no se refirió esta sospecha (ver Figura 3).

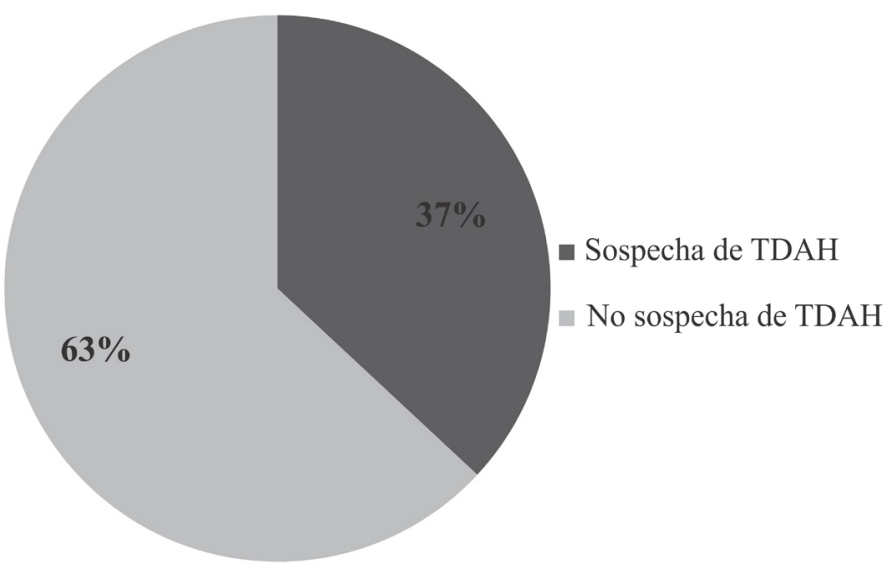

Figura 3. Distribución del porcentaje de los casos con y sin sospecha de TDAH por parte de los profesores.
Dentrodelanálisis estadístico X2 entre las variables estilo de crianza y sospecha de TDAH evaluado por parte de los padres, se obtuvo un valor $\mathrm{p}$ menor a $0.05(\mathrm{X} 2(2, \mathrm{n}=27)=6,750, \mathrm{p} 0.03)$. Observándose un aporte de $63 \%$ del valor X2 correspondiente a la relación entre el estilo de crianza autoritario y la no sospecha de TDAH referido por los padres. Señalando una dependencia significativa entre estas dos variables (ver Tabla 1).

Tabla1

Tabla de contingencia Estilo de crianza X Sospecha TDAH padres

\begin{tabular}{|c|c|c|c|c|c|}
\hline & & & specha $\mathrm{T}$ & $\mathrm{AH}$ padre & S Total \\
\hline & & & Sí & No & \\
\hline & Permisivo & $\%$ del total & $11,1 \%$ & $22,2 \%$ & $33,3 \%$ \\
\hline Estilo de crianza & Autoritario & $\%$ del total & $0,0 \%$ & $63,0 \%$ & $63,0 \%$ \\
\hline & Disciplinado & $\%$ del total & $0,0 \%$ & $3,7 \%$ & $3,7 \%$ \\
\hline Total & & $\%$ del total & $11,1 \%$ & $88,9 \%$ & $100,0 \%$ \\
\hline
\end{tabular}

En el análisis estadístico X2 para las variables estilo de crianza y sospecha de TDAH referidas por los profesores, se obtuvo un valor p mayor a 0.05 (X2 $(1, n=25)=0,011, p 0.91)$. Señalando que no existe dependencia significativa entre las variables (ver Tabla 2).

Tabla 2

Tabla de contingencia Estilo de crianza X Sospecha TDAH profesores

\begin{tabular}{|c|c|c|c|c|c|}
\hline & & & echa TD & H profesores & s $\quad$ Total \\
\hline & & & Sí & No & \\
\hline & Permisivo & $\%$ del total & $4,0 \%$ & $32,0 \%$ & $36,0 \%$ \\
\hline Estilo de crianza & Autoritario & $\%$ del total & $8,0 \%$ & $56,0 \%$ & $64,0 \%$ \\
\hline & Disciplinado & $\%$ del total & $0,0 \%$ & $0,0 \%$ & $0,0 \%$ \\
\hline Total & & $\%$ del total & $12,0 \%$ & $88,0 \%$ & $100,0 \%$ \\
\hline
\end{tabular}

\section{DISCUSIÓN}

Con base en los resultados obtenidos podemos observar respecto a la prevalencia de los estilos de crianza, una mayor presencia de padres que refieren tener un estilo de crianza autoritario para con sus hijos, datos que podrían sugerir un perfil característico del 
estilo de crianza para el grupo estudiado, considerando las condiciones propias sociodemográficas, aunque la muestra no es significativamente representativa para inferir sobre la población del municipio de Guadalajara, considerando que no existen datos que indiquen una tendencia sobre un estilo en particular que caracteriza a la población mexicana, nuestros datos podrían servir como referencia para futuras investigaciones que pretendan esta caracterización con el acceso a una muestra mayor.

Respecto a la prevalencia de las conductas asociadas al diagnóstico del TDAH los datos parecen coincidir con los de investigaciones como la de Cáceres y cols. (2011) en la cual los profesores muestran un mayor número de casos considerados con presencia del trastorno a diferencia de los padres, con respecto a nuestros datos se observó una diferencia del $26 \%$ entre el número de casos reportados por los padres y el número de casos reportados por los profesores, lo cual podría asociarse al escenario en el cual se presentan las conductas, difiriendo entre las ejercidas en casa y las ejercidas en la escuela, criterio a tomar en consideración para el adecuado diagnóstico del TDAH según la literatura (De la Peña, Palacio y Barragán, 2007; APA, 2013).

El análisis entre el cruce de variables de igual forma muestra datos contrariados, mientras que la relación entre los estilos de crianza y la apreciación por parte de los padres resulta significativa estadísticamente, la relación entre estilos de crianza y la apreciación por parte de los profesores no lo es. La prueba estadística aplicada nos arroja un valor significativo para suponer que existe dependencia entre el estilo de crianza ejercido por los padres y las apreciaciones de estos mismos sobre las conductas asociadas al TDAH presentes en sus hijos, mostrándose un mayor aporte a esta dependencia la relación entre el estilo de crianza autoritario y la ausencia de TDAH. Esto podría sugerir que el ejercer un estilo de crianza que se caracteriza por el establecimiento de reglas cuya violación implica un castigo, restringe la expresión de conductas de desatención e inhibición, lo cual se refleja en un menor reporte de estas conductas por parte de los padres, por lo que podría esperarse que estos no juzgarán a sus hijos como niños con TDAH.
Por su parte la relación entre los profesores que evaluaron las conductas asociadas al TDAH en los niños y el estilo de crianza de sus padres parece no tener una dependencia estadísticamente significativa, se destaca que pese a no alcanzar la significancia estadística la relación entre el estilo de crianza autoritario y la no sospecha de TDAH aporta en gran proporción al estadístico obtenido, coincidiendo con los reportes realizados por los padres que presentaban un estilo de crianza autoritario.

Debido a que para el análisis estadístico se consideraron solo aquellos casos en los que se tenían ambos instrumentos completos, quedaron descartados algunos (39\%) cuyos cuestionarios de los estilos de crianza no fueron enviados por sus padres, pero si se tenía la evaluación del profesor respecto a sus conductas. La evasión de la autoevaluación por parte de los padres con respecto al estilo de crianza que ejercen con sus hijos podría interpretarse como una conducta de desinterés e indiferencia, características de un estilo de crianza permisivo según la clasificación empleada. De tal manera que realizando un análisis de los datos bajo el supuesto de que estos padres que no regresaron sus cuestionarios ejercen un estilo de crianza permisivo se obtuvo un valor $\mathrm{p}$ menor a $0.05(\mathrm{X} 2(1, \mathrm{n}=43)=6,659$, p 0.010). Observándose un aporte igual del $32 \%$ del valor de la X2 correspondiente tanto a la relación entre el estilo de crianza permisivo y la sospecha de TDAH referida por profesores, como a la relación entre el estilo de crianza autoritario y la no sospecha de TDAH, sugiriendo una dependencia significativa entre estas dos variables. Estos resultados parecen coincidir con los presentados por Raya, Herraruzo y Pino (2008) quienes identificaban la presencia de un estilo de crianza democrático como predictor de las conductas de hiperactividad en los niños.

De acuerdo con los resultados los datos muestran una dependencia estadísticamente significativa entre las variables estilo de crianza y comportamientos referidos por los padres, sugiriendo que el estilo de crianza autoritario se asocia con un reporte de ausencia de comportamientos relacionados con el TDAH referido por parte de los padres. 


\section{REFERENCIAS}

Almeida, L. (2005). Alteraciones anatómicofuncionales en el trastorno por déficit de atención con hiperactividad. Salud Mental, 28 (3), 1-12.

Almeida, L., Ricardo-Garcel, J., Prado, H. y Martínez, R. (2010). Alteraciones estructurales encefálicas en el trastorno por déficit de atención e hiperactividad: una actualización. Segunda parte. Salud Mental, 33 (1), 77-84.

American Psychiatric Association (APA). (2014). Manual diagnóstico y estadístico de los trastornos mentales (DSM-5), $5^{\text {a }}$ ed. Arlington: Panamericana.

Ardila, A., Gómez, E., Matute, E., Ostrosky, F., Pineda, D., Rosselli, M. (2012). NEUROPSI Atención y Memoria. México: Manual Moderno.

Barrio, V. y Roa, L. (2001). Adaptación del Cuestionario de Crianza Parental (PCRI-M) a población española. Revista Latinoamericana de Psicología, 33 (3), 329-341.

Baumrind, D. (1966). Effects of Authoritative Parental Control on Child Behavior. Child Development, 887-907.

Cáceres, J. y Herrero, D. (2011). Cuantificación y análisis de la concordancia entre padres y tutores en el diagnóstico del trastorno por déficit de atención con hiperactividad (TDAH): rompiendo el estigma. Rev Neurol, 52. 527-532

Calvo, M., Herrera, E. y Peyres, C. (2003). El trastorno por déficit de atención con hiperactividad desde un perspectiva actual. Orientaciones a padres y profesores. Rev. De Psicol. Gral. Y Aplic, 56 (1), 5-19.

Cardo, E., De Azua, B., Redondo, M., Riutort, L., Severa, M. y Vidal, C. (2011). Influencia de los diferentes criterios de diagnóstico y la cultura en la prevalencia del trastorno por déficit de atención/ hiperactividad. Rev. Neurol, 52 (1). 109-117.

Castellanos, F. y Acosta, M. (2011). Hacia un entendimiento de los mecanismos moleculares de los tratamientos farmacológicos del trastorno por déficit de atención/hiperactividad. Rev Neurol, 52 (1), 155-160.
Clarke, A., Barry, R., McCarthy, R., Selikowitz, M. (2001) EEG-defined subtypes of children with attention-deficit/hyperactivity disorder. Clinical Neurophysiology, 112, 2098-2105.

Miranda-Casas, A., Herrero, M., Colomer-Diago, C, y Rosello, B. (2011). Satisfacción de la vida de niños con trastorno por déficit de atención/ hiperactividad: estudio de posibles factores de riesgo y protección. Rev. Neurol, 52 (Supl1). 5119-5126

Cornelio-Nieto,J., Borbolla-Sala,M.,Gallegos-Dimas, A. (2011). Alteraciones electroencefalográficas en niños con trastorno por déficit de atención/ hiperactividad. Rev Neurol, 52 (1), 97-101.

Correas, J., Quintero, J., Quintero, F. (2009) Trastorno por Déficit de Atención e Hiperactividad a lo largo de la vida. Barcelona: Masson Elsevier.

De la Peña, F., Palacio, J., Barragán, E. (2010). Declaración de Cartagena para el Trastorno por Déficit de Atención con Hiperactividad (TDAH): rompiendo el estigma. Rev. Cienc. Salud, 8 (1), 93-98.

Escobar, E., Schmidt, G., Sardinas, S. (2011). Alteraciones electroencefalográficas y trastorno por déficit de atención. Rev Soc Bol Ped, 50 (1), 3-6.

Farré A., y Narbona, J. (2013) Escalas para la evaluación del trastorno por déficit de atención con hiperactividad (EDAH), $7^{\mathrm{a}}$ ed. España. TEA.

Galimberti, U. (2006). Diccionario de Psicología. México: Siglo veintiuno editores.

Heatherton T. y Wagner D. (2011) Cognitive neuroscience of self-regulation failure. Trends in Cognitive Sciences, 15 (3), 132-139.

Huasca, P. (2006) En México hay un mal diagnóstico del trastorno por déficit de atención. Disponible en: http://www.cronica.com.mx/notas/2006/257626. html.

IBM (2011). IBM SPSS Statistics for Windows, Version 20.0. Armonk, NY: IBM Corp.

Johnstone, S. y Clarke A. (2009) Dysfunctional response preparation and inhibition during a visual Go/Nogo task in children with two subtypes of 
attention-deficit hiperactivity disorder. Psychiatry Research, 166, 223, 237.

Mestre, M., Samper, P., Tur, A. y Díez I. (2001). Estilos de Crianza y desarrollo prosocial de los hijos. Rev. De Psicol. Gral y Aplic. 54 (4), 691-703.

Mulas, F., Gandía, R., Roca, P., Etchepareborda, M. y Abad,L. (2012) Actualización farmacológica en el trastornos por déficit de atención/hiperactividad: modelos de intervención y nuevos fármacos. Rev Neurol, 54 (3), 41-53.

Navarrete, L. (2011). Estilos de crianza y calidad de vida en padres adolescentes que presentan conductas disruptivas en el aula (Tesis de maestría). Chile: Universidad de Bío Bío.

Palacios, J. (1988). Las ideas de los padres sobre la educación de sus hijos. Sevilla: Instituto de Desarrollo Regional.

Ramírez, M. (2005). Padres y desarrollo de los hijos: prácticas de crianza. Estud. Pedagóg. 31 (2), 167177.
Raya, A., Herraruzo, J. y Pino, M. 2008. El estilo de crianza parental y su relación con la hiperactividad. Psicothema, 20 (4), 691-696.

Stassen, K. (2007). Psicología del desarrollo Infancia y adolescencia. España: Médica Panamericana.

Stern P. y Shalev, L. (2013). The role of sustained attention and display medium in reading comprehension among adolescents with ADHD and without it. Research in Developmental Disabilities, 34, 431-439.

Torío, S., Peña, J. y Rodríguez, M. (2008). Estilo educativos parentales. Revisión bibliográfica y reformulación teórica. Teor. Educ. 20, 151-178.

Vygotsky, L. S. (1986). Historia del desarrollo de las funciones psíquicas superiores. Obras Escogidas Tomo III. Comisión editorial para la edición de la lengua rusa.Academia de Ciencias Pedagógicas de la URSS. http://www.taringa.net/perfil/vigotsky.

Fecha de recepción: 25 de octubre de 2016

Fecha de aceptación: 19 de noviembre de 2016 